\title{
Software Platform Dedicated for In-Memory Computing Circuit Evaluation
}

\author{
Maha Kooli ${ }^{1}$, Henri-Pierre Charles ${ }^{2}$, Clément Touzet $^{1}$, Bastien Giraud ${ }^{1}$, Jean-Philippe Noël ${ }^{1}$ \\ ${ }^{1}$ Univ. Grenoble Alpes, CEA, LETI, F-38000 Grenoble \\ ${ }^{2}$ Univ. Grenoble Alpes, CEA, LIST, F-38000 Grenoble \\ maha.kooli@cea.fr
}

\begin{abstract}
This paper presents a new software platform, co-developed by research teams with expertises in memory design, and software engineering and compilation aspects, to dimension and evaluate a novel In-Memory Power Aware CompuTing (IMPACT) system for IoT. IMPACT circuit is an emerging memory that promises to save execution time and power consumption by embedding computing abilities. The proposed platform permits to manually convert a software application from conventional to IMPACT implementation using vector representation. The two implementations are then compiled on the Low Level Virtual Machine (LLVM) and traced in order to evaluate their performance in terms of timing and energy consumption. The results of emulating image-processing and secure applications on IMPACT system show a significant gain in the execution time and the energy consumption compared to a conventional system with an ARM Cortex $(\mathrm{R}-\mathrm{M} 7$ processor. The execution time can be reduced from $50 \mathrm{x}$ to $6145 \mathrm{x}$, depending on the application and the workload size. Furthermore, the gain of the energy consumption is about $12.6 \mathrm{x}$.
\end{abstract}

\section{CCS CONCEPTS}

- Computer systems organization $\rightarrow$ Heterogeneous (hybrid) systems;

\section{KEYWORDS}

In-memory Computing, Performance, Software Platform, LLVM

ACM Reference Format:

Maha Kooli ${ }^{1}$, Henri-Pierre Charles ${ }^{2}$, Clément Touzet ${ }^{1}$, Bastien Giraud $^{1}$, Jean-Philippe Noël ${ }^{1}$. 2017. Software Platform Dedicated for In-Memory Computing Circuit Evaluation. In Proceedings of RSP'17, Seoul, Republic of Korea, October 15-20, 2017, 7 pages. https://doi.org/10.1145/3130265.3130322

Permission to make digital or hard copies of all or part of this work for personal or classroom use is granted without fee provided that copies are not made or distributed for profit or commercial advantage and that copies bear this notice and the full citation on the first page. Copyrights for components of this work owned by others than ACM must be honored. Abstracting with credit is permitted. To copy otherwise, or republish, to post on servers or to redistribute to lists, requires prior specific permission and/or a fee. Request permissions from permissions@acm.org.

RSP'17, October 15-20, 2017, Seoul, Republic of Korea

(C) 2017 Association for Computing Machinery.

ACM ISBN 978-1-4503-5418-9/17/10 . .\$15.00

https://doi.org/10.1145/3130265.3130322

\section{INTRODUCTION}

Researchers are nowadays focusing on memory improvement in particular the growing speed gap between memory and computation unit known as the "memory wall" (Figure 1). One solution to reduce the data movement and the resulting power consumption consists in bringing a part of the memory (i.e., the caches) near to the processor. However, to optimize the data access time the cache sizes are reduced, which requires multi cache levels leading to significant increase of the chip area. Therefore, other solutions came up over the years to cover these problems. They can be grouped in the following techniques: Processing-In-Memory, In-Memory-Computing, Logic-In-Memory.

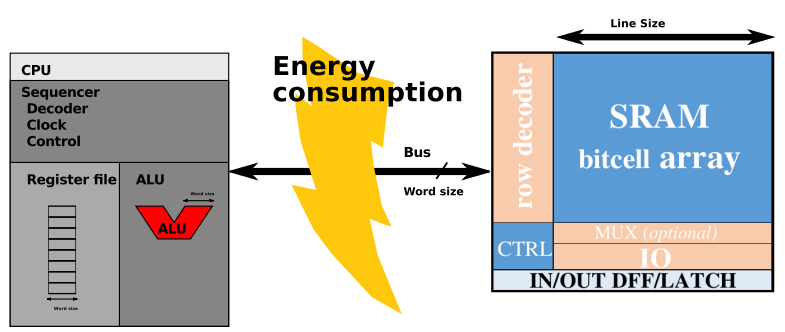

Figure 1: Conventional computing architecture which needs a memory access for all word level computation

Processing-In-Memory (PIM) is a process based on DRAM, which consists in putting the computation unit next to the memory while keeping the two dissociated [14]. The objective behind bringing the processor near to the memory aims to reduce the time required to access the data inside the memory and therefore the power consumption due to that. This concept has been first introduced in [6] and [17], where the memory and the CPU have been implemented in the same die. In more recent works and with the progress of the 3D technology, researchers propose to design stacks of computation unit (as CPUs [18] or as logic units [7]) next to the memory stack, which permits to create massive data parallelism. Logic-In-Memory is the concept of integrating some computation in the memory. However, it is more used to realize logic operations on a specific memory layer [16] or logic layer dedicated for 3Ds memories [21]. In-Memory Computing (IMC) consists in integrating a part of the computation units into the memory boundary (Figure 2), which means that the data do not go out from the memory. This should offer significant gain in the execution time and the 
power consumption. The IMC concept has been applied on volatile memories [8] and non volatile memories [15] [20] [10]. In [4], the authors present the architecture of in-memory computing applied on the caches. In [9], the authors discuss the different performance gain of this circuit for a face recognition algorithm. However, in all these papers, no software platform to simulate the in-memory computing circuit has been presented. All the applications that have been experimented to evaluate this concept, are manually analyzed. In this paper, we do not only focus on the architecture of this circuit, but also on the compilation and the programming flow, the point that has not been studied by the existing works.

\section{SRAM Architecture}

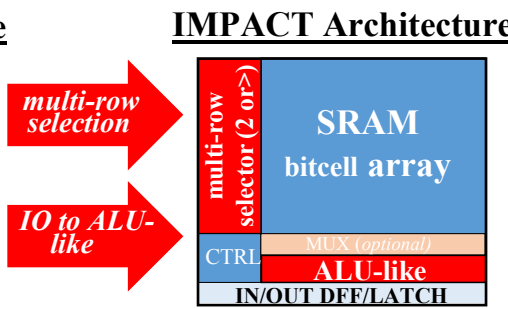

Figure 2: IMPACT circuit architecture derived from SRAM circuit architecture

In this article, we propose a new software platform that helps to design a new IMPACT circuit in order to quickly investigate different memory layout parameters under the light of real world and high level applications. Thanks to a high level instrumentation trace generator and a trace analyzer able to evaluate the performances. This study permits to help memory designers to select optimum configurations.

This paper is based on the work previously published in [5], where the concept of the IMPACT circuit has been first introduced. We present a novel software platform to rapidly prototype the IMPACT system and dimension its circuit. The objective of our research project is to: (i) prove a huge performance improvement in terms of execution time and energy consumption, (ii) help designer to select optimal configurations, and (iii) build a complete IMPACT system going from the software to the hardware layers (Figure 3). The proposed software platform permits to evaluate the execution time and the energy consumption of two software applications first run on a conventional IoT system and then simulated on IMPACT system. The first experimented application is a security one while the second is an image processing application. The results show a timing speed factor varying between 50 and 6145 times, and an energy gain of 12.6 times.

This paper is structured as follows. Section 2 presents a brief description of the IMPACT system. Section 3 introduces the software platform proposed to experiment the IMPACT circuit. Section 4 describes the experimentation. The scenarios and the results are then discussed in Section 5. Finally, Section 6 concludes the paper.

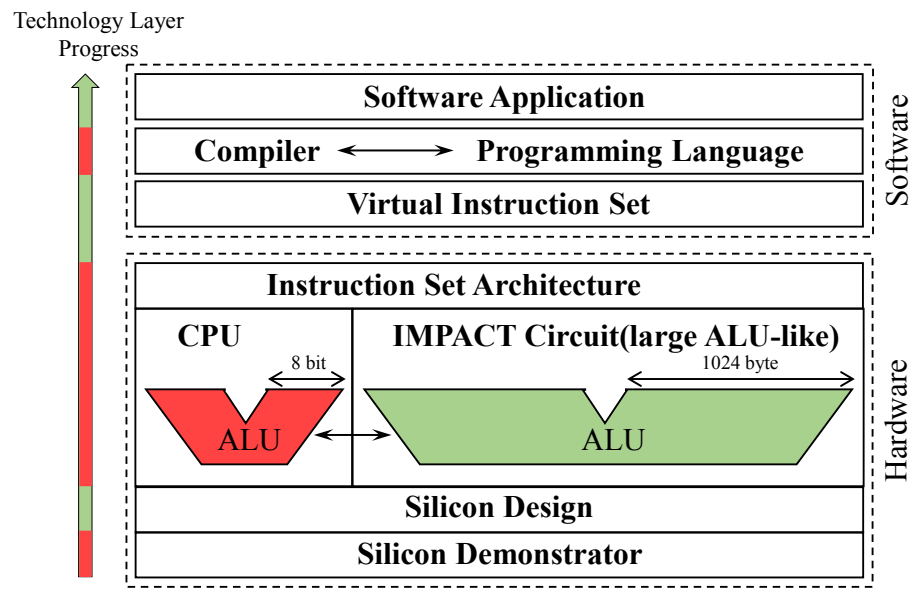

Figure 3: General project overview: Evaluating high level applications in order to design suitable memory architectures

\section{IMPACT SYSTEM DESCRIPTION}

In this section, we describe the concept of IMPACT Circuit.

The work published in [5] introduces the concept of IMPACT circuit. Based on efficient bitcells, it proposes a novel computing circuit using the SRAM architecture to enable inmemory logic and arithmetic operations, as shown in Figure 2. This permits to reduce the data transfer between the memory and the computing units (i.e., ALU). The proposed IMPACT circuit is able to perform the same logic and arithmetic operations as a basic ALU and, therefore enables non-destructive computing and storage in the same unit.

The IMPACT concept is based on an SRAM bitcell array in which multiple rows can be selected at the same time during the same clock cycle for performing in-situ operations between selected rows. The operation type is chosen and controlled slice-wise (using a dedicated controller), thus different operations can be performed on different slices of the same array offering highly-parallel operations. The IMPACT circuit is able to perform different arithmetic and logic operations inside the memory, thus limiting intensive data transfers.

Table 1 provides the description of the logic and arithmetic operations that the IMPACT memory is able to perform. The logic operations are performed in two clock cycles, while the arithmetic operations are performed in three cycles. Based on this timing, we compute the total number of cycles required to run an application on IMPACT system, as it will be presented in Section 5.

\subsection{Logic Operations}

A native logic operation can be performed when more than one row are selected in a bitcell column. Based on the tripleport SRAM bitcells enabling two read and one write operations per memory access cycle, the read-ports connected to the data stored in the write-port enable to perform NOR operations, while the ones connected to the complementary 
Table 1: Arithmetic and Logic Operations enabled by IMPACT System.

\begin{tabular}{|l|l|l|l|}
\hline $\begin{array}{l}\text { Operation } \\
\text { Type }\end{array}$ & Examples & $\begin{array}{l}\text { Cycles } \\
\#\end{array}$ & $\begin{array}{l}\text { Sub- } \\
\text { Operations* }\end{array}$ \\
\hline LOGIC & $\begin{array}{l}\text { OR, AND, } \\
\text { INV, XOR, } \\
\ldots\end{array}$ & 2 cycles & Rs -> Ws \\
\hline ARITHMETIC & $\begin{array}{l}\text { ADD, } \\
\text { INC, }\end{array}$ & DEC,, \\
& $\ldots$ & 3 cycles & $\begin{array}{l}\text { Rs -> L -> } \\
\text { Ws }\end{array}$ \\
\hline
\end{tabular}

* Rs: represents the load operation in IMPACT circuit

Ws: represents the store operation in IMPACT circuit

L: additional cycle for the logic execution

data enable to perform AND operations. Based on this behavior, an additional IO circuitry has been designed to perform the rest of valid logic operations with $\mathrm{N}$ operands ( $\mathrm{N}$ corresponding to the number of selected rows): OR, NAND, EQ (equal) and NEQ (not equal). A selection signal ADD enables to change the nature of the operation on the outputs of the circuitry, which is useful to differentiate addition and subtraction operations.

If only one row is selected, as in a conventional memory, the result on the true read bitline (RBLT), (resp. false read biltine) will be the value of the selected data (resp. complementary data). In this case, the resulting operations performed by the additional IO circuitry are: XOR, NXOR, INV and material implication. This operating mode is used to perform addition, subtraction and word-level comparison (greater than (GT), lower than (LT) and equal (EQ)) operations.

\subsection{Arithmetic Operations}

Based on the results of the logic operation of the additional IO circuitry, we implement a ripple-carry adder/subtractor. Only three logic gates are added to perform the operations which are usually implemented only with CMOS logic gates. The selection between the addition and subtraction operation is made by setting the appropriate value on the ADD signal (' 1 ' for addition, ' 0 ' for subtraction). Increment and decrement operations are performed in the same way by setting the carry to ' 1 '. All these operations can be performed in one time (e.g. in asynchronous operating mode) or in three equal cycles (e.g. in synchronous operating mode).

Other arithmetic operations are enabled by the IMPACT circuit, such as the comparison operation at word level (GT, LT and EQ). These operations are performed with the same timing constraints as the ADD/SUB operations by adding some logic gates to the additional IO circuitry.

\section{SOFTWARE PLATFORM DESCRIPTION}

This paper presents a preliminary software platform to emulate software applications on IMPACT circuit. The proposed platform will be used to: (i) demonstate a huge performance gain in terms of execution time and energy consumption for IoT, (ii) help designer to select optimal configurations and (iii) build a complete IMPACT system going from the software to the hardware layer.

In order to characterize a new compilation platform for IMPACT circuit, we need to investigate methods and tools to describe the software independently from the target hardware architecture. In fact, at this stage of our research, the Instruction Set Architecture (ISA) of the IMPACT hardware system (smart memory $+\mathrm{CPU}$ ) is not yet defined and implemented. Therefore, it cannot be used to analyze how the software applications is executed on the IMPACT system. In this paper, we make use of virtualization techniques, in particular LLVM [13], which is a compiler framework that uses virtualization with virtual instruction sets to perform complex analysis of software applications on different architectures. LLVM presents different characteristics that are advantageous for our tool. First, it uses the Intermediate Representation (IR) as a form to represent the code in the compiler, which is similar to an assembly code. The is independent from the source language and the target machine. Furthermore, LLVM allows code to be left for late-compilation from the IR to machine code via the just-in-time compilation. Then, LLVM is able to manipulate very large vectors (e.g., $<2048 \mathrm{x}$ i32> a vector of 2048 32-bit integer) and integer with arbitrary bit width varying from 1 bit to $2^{23}-1$ (about 8 millions) [3].

The proposed software platform consists in compiling the application implemented in two modes, the conventional and the IMPACT implementations. The IMPACT implementation is based on the LLVM vector representation of the data, where the word size is dynamically configured. This vector representation interprets the logic/arithmetic operations that are performed in IMPACT circuit. In fact, as explained in Section 2, this circuit is based on SRAM array in which multiple rows can be selected and computed at the same time.

In Figure 4, we provide the flow of the proposed platform going through different system layers. Starting from the $\mathrm{C}$ programming language, we implement the given software application in two versions: (1) the conventional version and (2) the smart version representing the behavior of the IMPACT memory, where the arithmetic/logic operations are computed on data represented as vectors. For example, as shown in Listing 1, a bit-wise between the elements of two byte arrays is implemented, in smart version, as a bit-wise between two vectors of bytes. Each vector corresponds to a line in the smart memory, and the operation is implemented using the LLVM capabilities to manage such operation on large integers/vectors. The output of the application is guaranteed to be the same for the two implementations. 


\section{Conventional System IMPACT System}

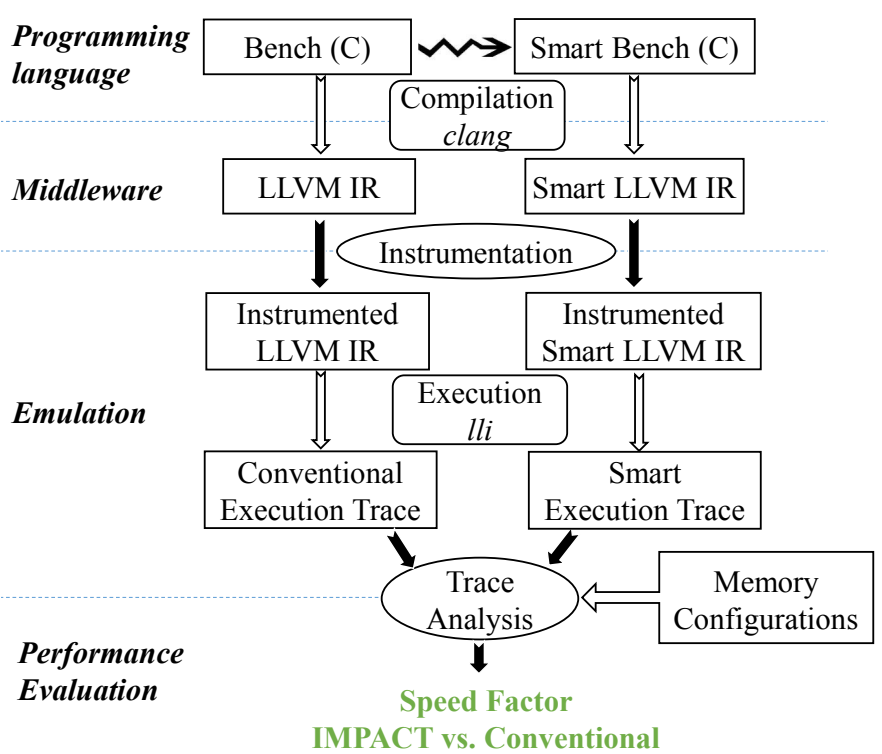

IMPACT vs. Conventional

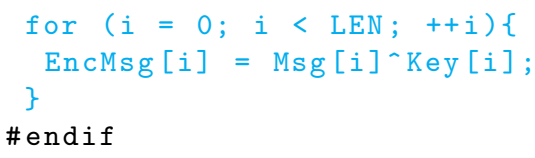

Listing 1: Conventional vs. IMPACT implementation

The traces resulting from the two executions are analyzed by computing the total number of cycles and the energy consumption. For the conventional execution, the number of cycle and the energy consuption are provided by the selected technology and processor. For the IMPACT execution, we consider the timing information provided in Table 1. The final step consists in computing the speed factor as the ratio between the cycles and energy consumption of the conventional execution and the IMPACT execution.

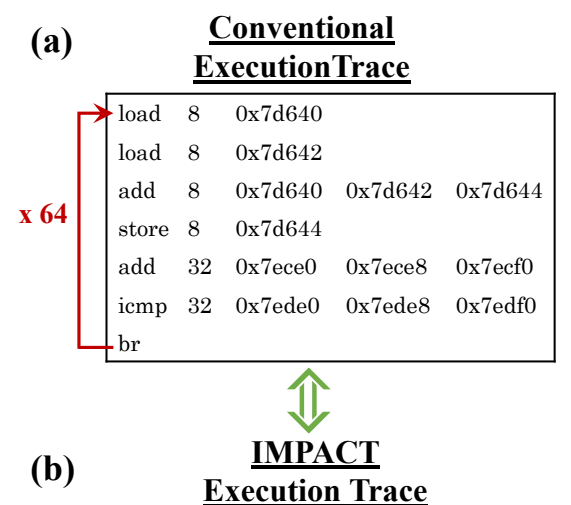

\begin{tabular}{|lllll|}
\hline smartAdd & 512 & 0x7d640 & 0x7d240 & 0x7ce40 \\
\hline
\end{tabular}

Figure 5: (a) Conventional execution trace, (b) IMPACT execution trace

\section{EXPERIMENTATION}

In order to evaluate the IMPACT circuit, we set up a conventional system for the sake of refrence. In this paper, we compare the execution time and the energy consumption required to simulate a software application on an IMPACT system to the execution time and the energy consumption required to run the same application on a conventional system. To have a fair comparison, we set up the same CPU for both systems. In particular, we use an ARM Cortex $(\mathrm{R}-\mathrm{M} 7$ processor, which is the most recent and highest performance member of the energy-efficient ARM Cortex-M series [1]. As shown in Figure 6, the conventional system is composed of a $\mathrm{CPU}$ (without cache to represent IoT devices) and an SRAM, while the IMPACT system is composed of a CPU and a smart memory based on SRAM.

To compute the timing of the conventional execution, we consider that the read/write operations take only one cycle to access data in the memory, and that the arithmetic/logic 


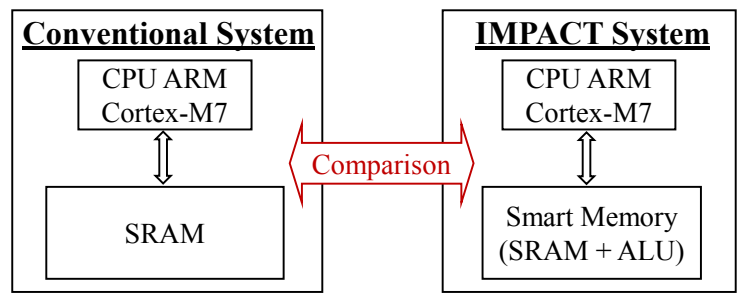

Figure 6: Experimental platform

operations take one cycle to be executed in the CPU. In fact, for this paper, we are considering the optimal case for the conventional system, where the chip frequency is equal to the memory frequency.

To compute the timing of the IMPACT execution, we use the information provided in Table 1 about the number of cycle required by each operation.

Then to evaluate the energy consumed by the application, we consider the energy consumed by each smart and conventional operation (read, write, arithmetic/logic). These information are provided thanks to measurements internally done in our research group.

Having the number of cycles and the energy consumed in pJ for each execution, we compute the gain of the IMPACT system comparing the conventional system. It is important to mention that for this paper, we evaluate the execution time and energy consumption only for the function that is performing the arithmetic/logic data computation. In addition, we assume that for the IMPACT memory, we have a single memory block where all the data are stored and computed.

\section{SCENARIOS AND RESULTS}

In this section, we evaluate the performances of IMPACT system emulating two software applications: (1) a security application presenting an input data of one dimension, and (2) an image processing application presenting an input data of two dimensions. For each scenario, we introduce the algorithms and discuss the results.

\subsection{One-Time Pad}

In cryptography, the One-Time Pad (OTP) [19] is an encryption technique that uses a private key generated randomly to encrypt a message. The message is then decrypted by the receiver using a matching one-time pad and key. In order to be efficient in terms of security (i.e., the message can not be decrypt or break), the key should be: truly random, at least as long as the message, never reused in whole or in part, and kept completely secret. OTP figured prominently in secret message transmission and espionage before and during the second World War and during the Cold War era.

In this paper, we make use of OTP as a preliminary application targeting security features. The algorithm is based on bit-wise operation between the secret key and the message, as shown in Algorithm 1. The operations used in this algorithm can be reused in the future to target more complex application scenarios targeting security.

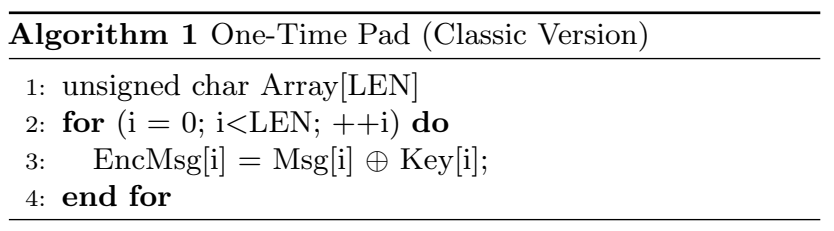

The algorithm is implemented in both conventional and IMPACT systems. In Listing 2, we present the LLVM IR code of the conventional implementation, where the encrypted message and the key are implemented as an array of characters, each character is of 1-byte long. The 'xor' is then performed byte per byte on the characters of the array. The complexity of the memory access is then linear $(\mathrm{O}(\mathrm{N}))$. However, for the IMPACT implementation, the encrypted message and the key are implemented as vectors, as shown in Listing 3 . The 'xor' is then performed on the vectors. The complexity of the memory access is then constant $(\mathrm{O}(1))$.

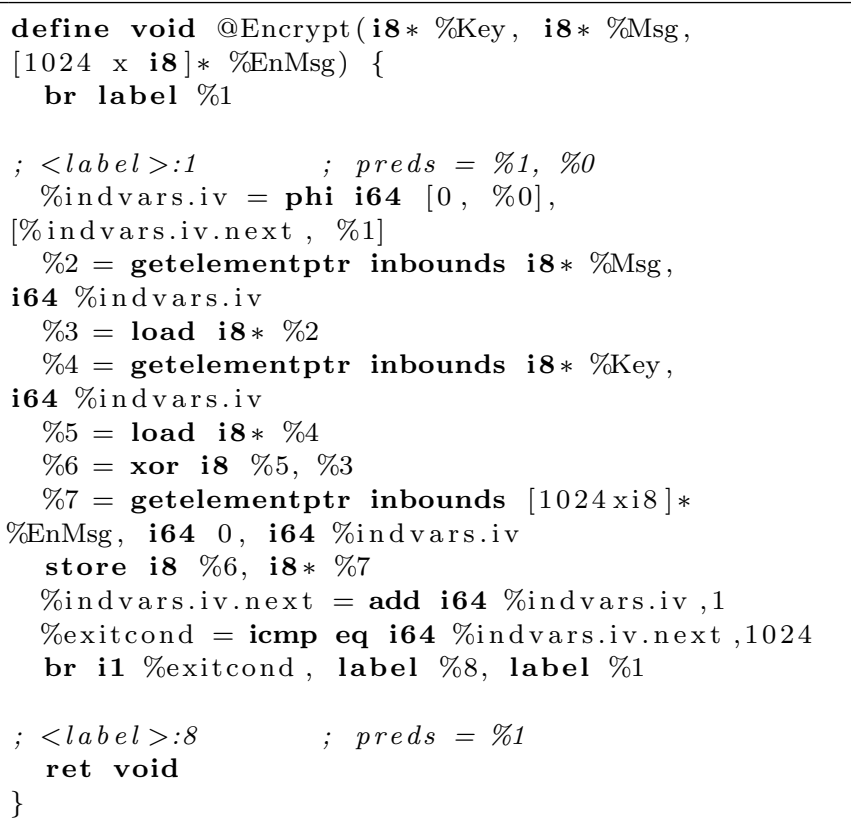

Listing 2: LLVM IR code of OTP application run on conventional system

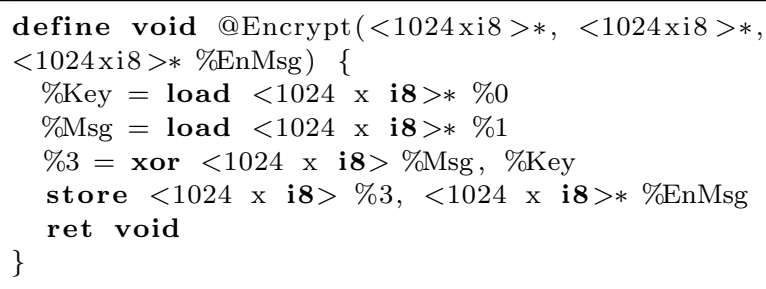

Listing 3: LLVM IR code of OTP application simulated on IMPACT system 
For the experiments, we consider, as workloads, different key sizes: 64, 128, 256, 512 and 1024 byte. In Figure 7, we present the timing speed factor of the IMPACT system compared to the conventional one. The acceleration of IMPACT emulation varies between $193 \mathrm{x}$ and $6145 \mathrm{x}$ times. On the other hand, the gain in the energy consumption is about $12.9 \mathrm{x}$ times the energy consumed by conventional system. The energy reduction does not vary when the workload data size change, since it is measured by words of data.

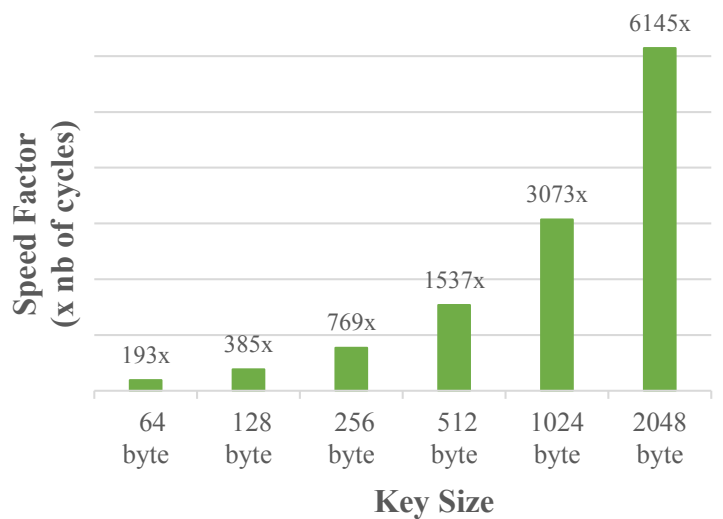

Figure 7: Timing speed factor between conventional and IMPACT systems for OTP application

\subsection{Motion Detection}

Motion detection [11] has attracted a great interest from computer vision researchers due to its promising applications in many areas, such as video surveillance, traffic monitoring or sign language recognition. Motion detection represents the basis of any object tracking, or image compression system. It is defined as the process of detecting a change in the object position relative to its surroundings or a change in the surroundings relative to an object.

The motion detection algorithm used in this paper consists in comparing the pixels of the image sequence frame in order to detect (or not) a movement in a certain zone of the image. As shown in Algorithm 2, the comparison of the images is performed by subtraction operation. The pixel colors of the resulting image represent the degree of difference between the two images.

The algorithm is implemented as conventional and IMPACT implementations. For the conventional version, the image is implemented as a matrix of pixels, each pixel is of 1 byte. The subtraction is then performed byte per byte on the matrix pixels. The complexity of the memory access is quadratic $\left(\mathrm{O}\left(N^{2}\right)\right)$. However, for the IMPACT version, the image is implemented as an array of vectors. The vectors represent the lines of the image pixels. The subtraction is then performed vector per vector. The complexity of the memory access is then linear $(\mathrm{O}(\mathrm{N}))$.

For the experiments, we consider images with different sizes:

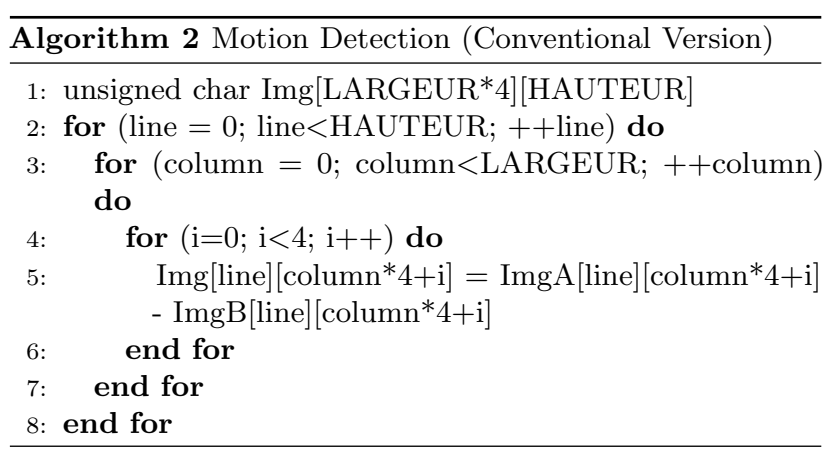

- X264 Standard of video compression: block sizes of $16 \times 16$ and $8 \times 8$ pixels.

- Graphics Display Resolution: 160x120 QQVGA, 320x240 QVGA, 640x480 VGA.

- High Definition: 960x540 qHD.

In Figure 8, we present the clock cycle speed factor of the IMPACT system compared to the conventional one. The results show that the acceleration varies between $50 \mathrm{x}$ and $5952 \mathrm{x}$ times the timing of the conventional system. The gain in the energy consumption is about $12.4 \mathrm{x}$ times the energy consumed by the conventional system. The energy reduction does not vary when the workload data size change, since it is measured by words by data.

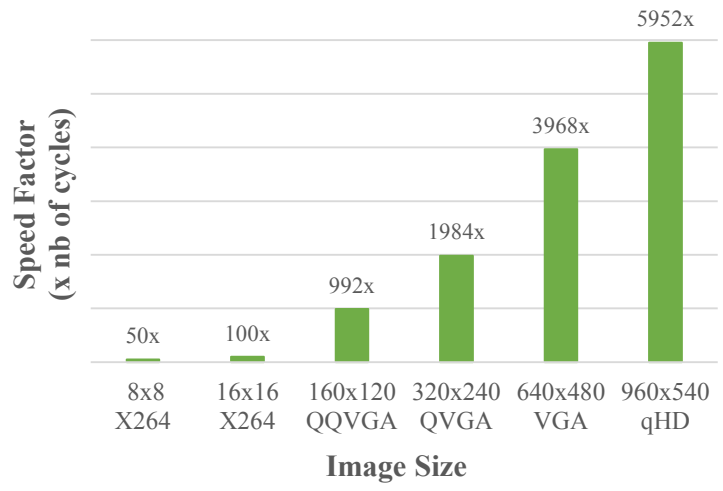

Figure 8: Timing speed factor between conventional and IMPACT systems for motion detection application

\subsection{Discussion}

We note from the presented results that the timing acceleration is more important for larger application workloads. Since we assume in this paper that the IMPACT memory is designed as single block, we have to design very large size IMPACT memories to run big data applications, which leads to increase the timing acceleration of the IMPACT memory. However, this is quite costly in particular for IMPACT circuit that are designed to be more complex than conventional circuits since it contains additional circuits that are responsible to perform operations. A possible solution that we envisage 
as a perspective to this work is to partition and design several IMPACT memory blocks with reasonable word size, then distribute the data workload on these blocks in a way to maximize the parallel operations. For example, the memory blocks can be superposed in 3D. An operation in one block can be then performed in parallel as an other operation in a second block. In particular, we can simulate the motion detection application on images of type VGA, using four images of type quarter VGA (QVGA) distributed on four memory blocks. The timing acceleration will be significant thanks to the parallelization, while the cost of the memory design will be reduced.

We note also from these results that the IMPACT system offers significant timing accelerations while less significant gain in the energy consumption. In fact, the energy study presented in this paper is preliminary. We need to investigate more effort to have more precise and significant gains in energy consumption, while keeping important timing accelerations.

As mentioned previously in Section 4, the acceleration factors shown in Figure 7 and 8 represent the execution time of the function that is performing the arithmetic/logic data computation. It will be interesting, in future work, to consider the rest of the application and also the rest of the system. The speed factor will be reduced relatively while remaining significant compared to a conventional system.

\section{CONCLUSION}

In this paper, we presented a new software platform able to dimension and evaluate our IMPACT circuit. The proposed approach permits to manually convert a software application from conventional implementation to IMPACT implementation, using an equivalent vector-parallel representation. The two implementations are then compiled to the middleware LLVM IR and instrumented in order to record traces of each execution. The traces are then analyzed to evaluate the performance of IMPACT circuit in terms of timing and energy consumption. In this paper, we present two application scenarios to experiment the IMPACT circuit: (1) a security application presenting input data of one dimension, and (2) an image processing application presenting input data of two dimensions.

The results of emulating these applications on IMPACT circuit show a significant gain in the execution time and a quite important gain in the energy consumption comparing to a conventional system run on an ARM Cortex $\mathbb{R}-\mathrm{M} 7$ processor. The timing acceleration varies between $50 \mathrm{x}$ and $6145 \mathrm{x}$, while the energy speed factor is about $12.6 \mathrm{x}$.

As perspective, several ideas can be developed. First, we propose to investigate effort to have more important and precise energy gain. Furthermore, we propose to partition the IMPACT memory in different blocks with small word size in order to parallelize the execution of operations, which should improve the system performance.

\section{REFERENCES}

[1] [n. d.]. ARM: Architecture for the Digital World, CortexM7 Processor. www.arm.com/products/processors/cortex-m/ cortex-m7-processor.php. ([n. d.]).

[2] [n. d.]. clang: a C language family frontend for LLVM. www.clang. llvm.org/. ([n. d.]).

[3] [n. d.]. LLVM Language Reference Manual. www.llvm.org/docs/ LangRef.htmlinteger-type. ([n. d.]).

[4] Shaizeen Aga et al. 2017. Compute Caches. In IEEE International Symposium on High Performance Computer Architecture (HPCA). 481-492.

[5] Kaya Can Akyel et al. 2016. DRC 2: Dynamically Reconfigurable Computing Circuit based on memory architecture. In IEEE International Conference on Rebooting Computing (ICRC). 1-8.

[6] Maya Gokhale et al. 1995. Processing in memory: The Terasys massively parallel PIM array. Computer 28, 4 (1995), 23-31.

[7] Joe Jeddeloh and Brent Keeth. 2012. Hybrid memory cube new DRAM architecture increases density and performance. In Symposium on VLSI Technology (VLSIT). 87-88.

[8] Supreet Jeloka et al. 2016. A $28 \mathrm{~nm}$ Configurable Memory (TCAM/BCAM/SRAM) Using Push-Rule 6T Bit Cell Enabling Logic-in-Memory. IEEE Journal of Solid-State Circuits 51, 4 (2016), 1009-1021.

[9] Dongsuk Jeon et al. 2017. A 23-mW Face Recognition Processor with Mostly-Read 5T Memory in 40-nm CMOS. IEEE Journal of Solid-State Circuits 52, 6 (2017), 1628-1642.

[10] V Joshi et al. 2017. Low-variation SRAM bitcells in 22nm FDSOI technology. In Symposium on VLSI Technology. IEEE, T222T223.

[11] Kamna Kohli and Jatinder Pal Singh. 2013. Motion Detection Algorithm. International Journal of Computer Science \&S Applications (TIJCSA) 1, 12 (2013).

[12] Maha Kooli. 2016. Analysing and Supporting the Reliability Decision-making Process in Computing Systems with a Reliability Evaluation Framework. Theses. Université Montpellier.

[13] Chris Lattner and Vikram Adve. 2004. LLVM: A compilation framework for lifelong program analysis \& transformation. In Proceedings of the international symposium on Code generation and optimization: feedback-directed and runtime optimization. IEEE Computer Society, 75.

[14] Dominique Lavenier et al. 2016. DNA Mapping using Processor-inMemory Architecture. In Workshop on Accelerator-Enabled Algorithms and Applications in Bioinformatics. Shenzhen, China.

[15] Shuangchen Li et al. 2016. Pinatubo: A processing-in-memory architecture for bulk bitwise operations in emerging non-volatile memories. In 53nd ACM/EDAC/IEEE Design Automation Conference $(D A C)$. 1-6.

[16] Shoun Matsunaga et al. 2009. MTJ-based nonvolatile logic-inmemory circuit, future prospects and issues. In Proceedings of the Conference on Design, Automation and Test in Europe. European Design and Automation Association, 433-435.

[17] David Patterson et al. 1997. A case for intelligent RA. IEEE Micro 17, 2 (1997), 34-44.

[18] Seth H Pugsley et al. 2014. NDC: Analyzing the impact of 3Dstacked memory + logic devices on MapReduce workloads. In IEEE International Symposium on Performance Analysis of Systems and Software (ISPASS). 190-200.

[19] Bruce Schneier. 2007. Applied cryptography: protocols, algorithms, and source code in C. john wiley \& sons.

[20] Yuhao Wang et al. 2016. DW-AES: A Domain-Wall NanowireBased AES for High Throughput and Energy-Efficient Data Encryption in Non-Volatile Memory. IEEE Transactions on Information Forensics and Security 11, 11 (2016), 2426-2440.

[21] Qiuling Zhu et al. 2013. A 3D-stacked logic-in-memory accelerator for application-specific data intensive computing. In $I E E E$ International 3D Systems Integration Conference (3DIC). 1-7. 